'Departamento de Educación Médica, Facultad de Medicina, Universidad de Concepción. Concepción, Chile.

angeniero Informático, Magíster en Educación Médica para las Ciencias de la Salud.

bPsicóloga, Magíster en

Ciencias de la Educación, mención Didáctica e Innovación Pedagógica.

cKinesióloga, Magíster en Educación Médica para las Ciencias de la Salud.

dBioquímica, Magíster en Educación Médica para las Ciencias de la Salud. ePsicóloga.

Fuente de apoyo Financiero: Proyecto VRID No 214.090.004-

10, Vicerrectoría de

Investigación, Universidad de Concepción. Apoyo financiero para la ejecución del Proyecto

"Dimensión didáctica de los escenarios educativos en carreras en Ciencias de la Salud".

La institución que proporcionó el financiamiento no tuvo influencia en el diseño del estudio; en la recolección, análisis o interpretación de los datos;

ni en la preparación, revisión o aprobación del manuscrito. Los autores declaran que no existen conflictos de interés.

Recibido el 20 de enero de 2017 , aceptado el 18 de julio de 2017.

Correspondencia a: Javiera Ortega B.

Chacabuco esquina Janequeo $S / N$ Concepción, Chile. javieraortega@udec.cl

\section{Condiciones del contexto educativo para ejercer el rol docente en Ciencias de la Salud. Un enfoque cualitativo}

\author{
OLGA MATUS B. ${ }^{1, a}$, JAVIERA ORTEGA B. ${ }^{1, b}$, PAULA PARRA P..$^{1, c}$, \\ LILIANA ORTIZ M. ${ }^{1}$, CAROLINA MÁRQUEZ U. ${ }^{1, d}$, \\ MELITA STOTZ R. ${ }^{1, \mathrm{e}}$, EDUARDO FASCE H. ${ }^{1}$
}

\section{The educational context to train health care professionals. A qualitative approach}

Background: There are differences in the educational context in Health Sciences, between clinical and non-clinical teachers. Therefore, the didactic and reflexive peculiarities of both educational scenarios should be analyzed. Aim: To describe the conditions of the educational context in Health Sciences for the practice of the teaching role in a Chilean university. Material and Methods: Qualitative study, performed according to Grounded Theory of Strauss and Corbin. Thirty one teachers from six health sciences programs were selected according to Patton's maximum variation criterion and contacted personally, after an informed consent process. Semi-structured interviews and focus group were performed, analyzed by open coding, using the constant comparison method, with the Atlas-ti 7.5.2 software. Results: Six conditions of the educational context that can support the teaching role in these careers emerged. Namely, a clinical field suited for patient attention and teaching, classrooms designed for the new educational models, number of students in the classrooms and clinical settings, insertion programs for teachers' training, teachers' coordination and economic resources of the program. Conclusions: Health Sciences programs are developed in a complex educational context, having to articulate diverse elements to train professionals. Therefore, it is fundamental to understand the conditions of the educational context that can favor the practice of the teaching role, thus generating improvements in teaching-learning process.

(Rev Med Chile 2017; 145: 926-933)

Key words: Grounded Theory; Health Education; Health Educators; Qualitative Research.
A ctualmente en las universidades se presta cada vez más atención a la calidad de la enseñanza. De acuerdo a la literatura, existen diversas definiciones que pretenden identificar las características de una enseñanza efectiva, considerando diversas perspectivas teóricas, con enfoques cuantitativos y cualitativos, desde varios puntos de vista disciplinares, de estudiantes y docentes. Según Devlin \& Samarawickrema la enseñanza efectiva se puede considerar una "ense- ñanza orientada y enfocada en los estudiantes y su aprendizaje, que requiere un conjunto particular de habilidades y prácticas, requisitos del contexto

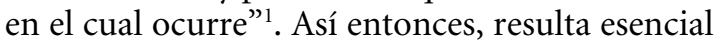
comprender cómo es el contexto educativo en el cual se realiza el proceso formativo.

La actividad docente es principalmente función de los profesores, quienes cumplen una importante función en la formación de los estudiantes, jugando un rol esencial para guiarlos en un conjunto de 
prácticas necesarias para ejercer su rol profesional en la sociedad ${ }^{2}$. El rol del docente es un medio para el aprendizaje de los estudiantes, lo que constituye el fin último de la educación, por lo que numerosos estudios declaran la importancia de que el aprendizaje debe centrarse en el estudiante ${ }^{1,3}$.

Históricamente, muchos filósofos y educadores han examinado el rol del docente y de las condiciones del contexto educativo. Platón, Confucius, Buber, Vigotsky y Freire coinciden en que la enseñanza no es una mera transmisión de conocimientos, sino más bien un aprendizaje mutuo $^{2}$. Por tanto, tan importante como el docente es el contexto educativo, que puede influenciar el aprendizaje de los estudiantes ${ }^{4,5}$.

Estudios realizados por Devlin y Samarawickrema $^{1}$ y por Badía y Gómez ${ }^{5}$ han identificado diversos factores involucrados en el contexto educativo: a) características de la institución educativa, incluyendo modelos de formación por competencias y de educación multiprofesional; b) el docente, para quien es importante la capacitación en docencia y los mecanismos de selección; c) características de los estudiantes, considerando sus estilos y estrategias de aprendizaje, y criterios de selección de la institución; d) naturaleza de la disciplina; e) cantidad de estudiantes por clase, relacionado con las necesidades de infraestructura para actividades en grupo pequeño y clases magistrales, en pos de un buen ambiente educacional; f) prácticas evaluativas, considerando evaluación sumativa, formativa y mecanismos de feedback; g) recursos materiales; y h) contexto clínico (considerando diversos tipos de campos clínicos) y social ${ }^{6}$. Estos factores están en constante interacción, pudiendo influir no solamente en el sentido de eficacia del profesor sino también en sus concepciones sobre la enseñanza, lo que puede condicionar el modo en que el docente desarrolla el diseño pedagógico de un curso $^{5,7-9}$. Las concepciones que pudieran influir en el análisis del contexto educativo pueden ser comparables a las creencias. Algunos autores las consideran entidades relativamente estables, mientras otros piensan que son dinámicas y dependientes del contexto $^{8,9}$. De hecho, se ha evidenciado que los estudiantes cuyos profesores tienen concepciones de alta eficacia, tienden a tener mejores resultados de aprendizaje $\mathrm{e}^{9,10}$.

Finalmente, desde Flexner, se han venido analizando las características del contexto educativo clínico y no-clínico de los programas de enseñanza de Ciencias de la Salud" ${ }^{11}$ La aparición del "Informe Flexner" en 1910, fue un hecho trascendental en la historia de la formación de médicos durante el siglo XX, incluyendo recomendaciones que hasta hoy son posibles de aplicar ${ }^{12}$. Flexner atribuyó un importante rol a las disciplinas académicas correspondientes a las ciencias básicas biomédicas en el desarrollo del pensamiento científico vinculado al razonamiento clínico de los médicos. Los avances y críticas a su modelo pudieran derivarse de la parcial aplicación de sus recomendaciones ${ }^{12,13}$. En los 100 años transcurridos desde su informe, el mundo, el objeto de estudio de las Ciencias de la Salud y la misión del médico han cambiado y por ello existen diversos enfoques acerca de la vigencia de su pensamiento. Uno de ellos es el de Schön ${ }^{14}$, quien postuló que a partir de este informe las facultades de medicina han estructurado su currículum de forma que han provocado una suerte de dualismo en la formación profesional.

Por lo anterior, el objetivo de este estudio fue describir las condiciones del contexto educativo para ejercer el rol docente en carreras del área de la salud en una Universidad de alta complejidad.

\section{Material y Método}

Investigación cualitativa, basada en la Teoría Fundamentada de Strauss y Corbin ${ }^{15}$. Los participantes fueron 30 docentes de 6 carreras de las Ciencias de la Salud: Medicina, Enfermería, Kinesiología, Fonoaudiología, Tecnología Médica y Obstetricia y Puericultura de una Universidad tradicional chilena y un experto en didáctica de profesión pedagogo (Tabla 1).

\section{Tabla 1. Distribución de Informantes por carrera}

\begin{tabular}{|lcc|}
\hline Profesión & n & $(\%)$ \\
\hline Enfermero (a) & 4 & $(12,9)$ \\
\hline Fonoaudiólogo(a) & 5 & $(16,1)$ \\
\hline Ingeniero & 1 & $(3,2)$ \\
\hline Kinesiólogo (a) & 3 & $(9,7)$ \\
\hline Matrón (a) & 6 & $(19,4)$ \\
\hline Médico cirujano & 6 & $(19,4)$ \\
\hline Profesor (a) & 1 & $(3,2)$ \\
\hline Tecnólogo médico & 5 & $(16,1)$ \\
\hline Total & 31 & $(100)$ \\
\hline
\end{tabular}


Los sujetos fueron seleccionados según el criterio de máxima variación de Patton ${ }^{16}$ hasta alcanzar la saturación de datos. Los informantes claves fueron los jefes de las carreras estudiadas, previo proceso de consentimiento informado institucional y de la carrera. Los participantes tenían una edad media de 43,6 años (D.E = 12,4), con mínimo de 26 años y máximo de 69 . De éstos, 38,7\% $(\mathrm{n}=12)$ eran hombres y $61,3 \%(\mathrm{n}=19)$ mujeres. $19,4 \%$ $(\mathrm{n}=6)$ de los entrevistados impartían docencia clínica, $48,4 \%(\mathrm{n}=15)$ docencia en aula y $32,3 \%$ $(\mathrm{n}=10)$ en contextos mixtos aula/clínica.

Las técnicas de recolección de información fueron entrevista semi-estructurada ${ }^{17}$ y focus group, grabados en audio y analizados mediante el método de comparación constante, hasta llegar al nivel de codificación abierta ${ }^{15}$. Se realizaron los análisis en el caqdas Atlas-ti 7.5.2. El estudio fue aprobado por el Comité de Ética de la Universidad ejecutora, visado por la Vicerrectoría de Investigación y Desarrollo de ésta.

\section{Resultados}

Como resultado del análisis de la codificación abierta, surgen 6 categorías emergentes asociadas a condiciones del contexto educativo, que pueden apoyar el rol docente en carreras de las Ciencias de la Salud, según los participantes en el estudio.

Las categorías se describen a continuación, siendo ilustradas mediante viñetas (citas textuales) en la Tabla 2. Cada viñeta es acompañada de un número arábigo que identifica su informante, indicándose además su sexo, disciplina y edad.

\section{Tabla 2. Categorías emergentes asociadas a condiciones del contexto educativo}

\begin{tabular}{|c|c|}
\hline Categoría & Viñeta ilustrativa \\
\hline \multirow{4}{*}{$\begin{array}{l}\text { Campo clínico } \\
\text { docente-asistencial } \\
\text { como contexto } \\
\text { natural del } \\
\text { proceso de } \\
\text { enseñanza- } \\
\text { aprendizaje }\end{array}$} & $\begin{array}{l}\text { "...los campos clínicos son indiscutibles y uno aprende, aprende en la práctica. Porque es (...) una pro- } \\
\text { fesión en la que tú tienes que pasar de tu cerebro a tus manos, a lo práctico, o sea el, el impacto real } \\
\text { de la cosa. Porque no saco ni nada si enseño en la sala y no salgo a hacer nada afuera..." [Informante } \\
\text { 11, Hombre, Kinesiología, } 69 \text { años] }\end{array}$ \\
\hline & $\begin{array}{l}\text { "...es algo bueno que tiene la carrera, que tiene un muy buen sistema de campo clínico. Entonces nos } \\
\text { permite potenciar esa parte (...) de enseñar por medios más que nada de demostraciones, de prácticas } \\
\text { clínicas, de ese tipo de cosas..." [Informante 1, Mujer, Tecnología Médica, } 31 \text { años] }\end{array}$ \\
\hline & $\begin{array}{l}\text { "...tus colegas que no hacen docencia, esperas que de cierta manera te den esas oportunidades para } \\
\text { el alumno. Entonces, ahí (...) hay colegas que de repente te llevas muy bien con ellos, y te permiten y } \\
\text { te dan estas oportunidades, pero de repente tú tienes colegas que les gusta hacer las cosas ellos, para } \\
\text { evitar errores que (...) porque son más rápidas o porque hay cierto temor a que pueda pasar algo, o } \\
\text { porque realmente no les gusta..." [Informante 5, Mujer, Obstetricia y Puericultura, } 28 \text { años] }\end{array}$ \\
\hline & $\begin{array}{l}\text { "...entonces eso [la cantidad de estudiantes en la clínica] te produce también un estrés en la sala que } \\
\text { están alumnos de enfermería, además los ocho de medicina, más los que están estudiando para técnico } \\
\text { paramédico, más los docentes, más los papás, las visitas, entonces la sala con ocho niños se convierte } \\
\text { como si tu sacas la cuenta como treinta y dos personas extra aparte de los niños..." [Informante 3, } \\
\text { Mujer, Enfermería, } 44 \text { años] }\end{array}$ \\
\hline \multirow{3}{*}{$\begin{array}{l}\text { Contexto } \\
\text { educativo en } \\
\text { aula como } \\
\text { espacio inicial } \\
\text { del aprendizaje } \\
\text { clínico }\end{array}$} & $\begin{array}{l}\text { "Como siempre estoy en aula (...) hay material eh, disponible de TIC's digamos disponibles. Siempre } \\
\text { hay un computador, un data" [Informante 2, Mujer, Obstetricia y Puericultura, } 45 \text { años] }\end{array}$ \\
\hline & $\begin{array}{l}\text { "A sala de clase es grande, se sientan dónde quieren, uno está adelante hablando y ellos están sentados } \\
\text { o ellos están hablando exponiendo y uno está sentado y no se dan esos tiempos de conversación. Y eso } \\
\text { del laboratorio es bien agradable y eso es bien normal en el fondo, de un laboratorio de hospital (...) } \\
\text { que se dan esos, esos espacios para conversar y para hacer más vida social que ambientes de trabajo" } \\
\text { [Informante 6, Mujer, Tecnología Médica, } 31 \text { años] }\end{array}$ \\
\hline & $\begin{array}{l}\text { "En la facultad (...) las salas son muy estructuradas. Esto de tener los mesones fijos no te permite hacer } \\
\text { otro tipo de trabajo más que de repente, ya tú quieres hacer un trabajo grupal en sala (...) me las he } \\
\text { arreglado para que traigan las sillas y las pongan tres adelante, tres al lado para que hagan grupos de } \\
\text { seis, pero es, es complicado. No está pensado para eh, los nuevos tiempos las salas. No ayudan mucho } \\
\text { en eso pero tienen buena luminosidad. No tienen buena extracción de aire pero son muy chicas para } \\
\text { la cantidad de alumnos que tenemos" [Informante 2, Mujer, Obstetricia y Puericultura, } 45 \text { años] }\end{array}$ \\
\hline
\end{tabular}




\section{Tabla 2. Categorías emergentes asociadas a condiciones del contexto educativo (continuación)}

Categoría
Valoración del
proceso de
selección y perfil
de los estudiantes
que ingresan a las
carreras de la salud

Cantidad de estudiantes como condición de la gestión en el proceso de enseñanzaaprendizaje

\section{Viñeta ilustrativa}

"... aquí hay diferentes [características de los alumnos], o sea de partida está el alumno que no me interesa (...) ese es el peor, que no le interesa nada, aunque no sean muchos pero existen. Está la mayoría que son alumnos que les interesa pero no saben (...) Y hay un grupito de alumnos que, que les interesa y saben..." [Informante 14, Hombre, Médico, 49 años]

"... una evaluación que te diga que tu no importa si de eso dependa la evaluación pero que el alumno le quede claro lo que lo que uno pide o lo que, o lo que intentaría que tuvieran una aspecto actitudinal para que el día de mañana no llegue la sorpresa (...) que le cuesta hablar en público o le molesta la gente ponte tu..." [Informante 1, Mujer, Tecnología Médica, 31 años]

"...uno no los conoce fuera de que haya algún alumno específico que tú puedas conocer con alguna característica especial o porque se ha acercado a ti. Es difícil conocerlos, uno los conoce más bien en el segundo semestre cuando tú tienes estos grupitos chicos. Que tienes uno o dos grupos, entonces conoces a esos diez alumnos... " [Informante 13, Mujer, Enfermera, 68 años]

"...necesitamos como mayor capacidad docente para que el grupo tampoco sea muy grande y tu puedas como supervisarlos bien a todos. Y más que supervisarlo, estar con ellos, acompañarlos para que ellos se sientan seguros, porque es distinto estar con el docente al lado, que si tu tienes alguna duda, que si tu tienes algún problema, que él te pueda ayudar en el fondo a cómo solucionar el problema..." [Informante 1, Mujer, Tecnología Médica, 31 años]

"...en clínica se debe monitorear a todos los evento activos también, o sea, el profesor, el estudiante, el paciente y también al equipo, porque el equipo frente a sus acciones puede generar también eventos que uno pueda rápidamente controlar o rápidamente zanjar... " [Informante 10, Fonoaudiología, años]

Formación docente en educación médica como eje principal en la calidad educativa

Recursos económicos y espacio físico para asegurar calidad en educación "...eso sería una súper buena política, o sea yo se que en otra universidad un docente que ingresa tiene que tener (...) una pincelada en educación. Es como obligatorio casi, pero acá no sé si la palabra obligatorio todavía está. Yo creo que se les sugiere..." [Informante 3, Mujer, Enfermería, 44 años]

"Yo llegué como docente (...) en ese semestre en realidad apliqué la docencia en base a un modelo que, que tenía cuando yo era estudiante. El como lo hacían mis docentes. Y después hice el diploma de educación médica y me entregó muchas herramientas para saber aplicar de forma más efec.va la docencia. Entonces me empezó, me ayudó a entender qué eran los resultados del aprendizaje, me ayudó a planificar una clase, ¿ya? y me ayudó a entender de que hay un proceso de enseñanza aprendizaje que es distinto en todos los alumnos y dependiendo de la etapa en que cada uno los toma va entendiendo que actividades van a ir favoreciendo el aprendizaje de este alumno o no. Y me ha enseñado que una de las cosas más importantes de la evaluación es la retroalimentación" [Informante 8, Hombre, Obstetricia y Puericultura, 32 años]

"No me parece que un docente esté por tercera vez repitiendo lo mismo, para poder enseñarlo porque y en condiciones deplorables desde un punto de vista físico. En un subterráneo, donde no hay ventilación, donde está todo o cerrado, donde está ahí las salas con diez camillas, donde se están chocando unos con otros. Entonces, no hay una, sincronización, digamos, entre espacio físicos a veces enseñanza, el profesor y los objetivos educativos" [Informante 11, Hombre, Kinesiólogo, 69 años]

"...entonces ahora con este laboratorio ellos [podría tener], quedarían mucho mejor preparados como para enfrentarse y trabajar en cualquier parte..." [Informante 4, Mujer, Tecnología Médica, 58 años]

"...la universidad como institución digamos en, en su jerarquía como superior siempre nos ha apoyado para, para el desarrollo. Pero es un, es un apoyo verbal ¿ah? pero de ahí a que se traduzca en, en, en lo que nosotros necesitamos, en la parte operacional, que necesitamos más oficinas, más computadoras, más, más modernitos, porque aquí si tu no cambias un computador cada diez años es mucho..." [Informante 13, Mujer, Enfermería, 68 años] 


\section{Campo clínico docente-asistencial como contex-} to natural del proceso de enseñanza-aprendizaje El campo clínico en carreras de Ciencias de la Salud cumple una función primordial en el proceso de aprendizaje y desarrollo de competencias práctico-clínicas. Por ende, estas carreras necesitan contar con campos clínicos apropiados para que los estudiantes puedan vincular el aprendizaje teórico con el práctico.

$\mathrm{Al}$ respecto, los docentes valoran que la institución cuente con campos clínicos importantes, resaltando que esto permite potenciar el desarrollo del razonamiento clínico, competencia transversal en todas estas carreras.

Considerando lo anterior, los docentes destacan facilitadores y obstaculizadores de los campos clínicos. La variedad de pacientes, áreas de especialidad en las carreras y contar con profesionales que apoyen el proceso de enseñanza clínica son facilitadores. Sin embargo, la predisposición de los profesionales no docentes pudiese ser un obstaculizador, si éstos no confían en los estudiantes que llegan a la clínica.

Otro aspecto obstaculizador son los espacios físicos de los servicios clínicos, no habilitados para recibir gran cantidad de estudiantes por carrera, dificultando el proceso de gestión de la docencia clínica y produciendo estrés en estudiantes, docentes, profesionales no docentes, pacientes y sus familias.

\section{Contexto educativo en aula como espacio ini- cial del aprendizaje clínico}

Los docentes mencionan que, para realizar un proceso educativo adecuado al nuevo modelo de enseñanza basado en competencias, es necesario contar con salas de clases aptas para la formación profesional, que les permita desarrollar sus actividades de enseñanza de manera adecuada y efectiva.

En la mayoría de las asignaturas, los docentes guían el proceso de aprendizaje de contenidos teóricos en salas de clases, a través de clases magistrales. En este contexto, los alumnos pueden sentarse donde desean, lo que puede facilitar o dificultar el proceso de aprendizaje, a diferencia de lo que ocurre en el contexto de laboratorio o clínico.

Un obstaculizador en el contexto aula, surge cuando las salas de clases no están ambientadas a las nuevas metodologías de enseñanza, requiriendo que los docentes deban adaptar algunas metodologías de enseñanza o bien solicitar a los alumnos que ayuden a conseguir materiales o sillas.

\section{Valoración del proceso de selección y perfil de} los estudiantes que ingresan a las carreras de la salud

Debido al proceso de selección, existen diversos perfiles de estudiantes en las carreras del área de la salud, como los estudiantes que no tienen deseo por aprender, aquellos que les interesa participar en el proceso de aprendizaje, pero no logran los resultados esperados y aquellos que se orientan a aprender y lo logran exitosamente.

El proceso de selección universitaria chilena utiliza un único instrumento para determinar cuáles estudiantes ingresan a las instituciones de Educación Superior. Esto ha permitido a la Universidad de Concepción contar con estudiantes que obtienen altos puntajes en la prueba de ingreso. Sin embargo, algunos docentes señalan que sería fundamental incluir instrumentos de evaluación que permitieran identificar otras habilidades de los estudiantes que son vitales en carreras de la salud, como sus habilidades de relación con pacientes y equipos de trabajo.

\section{Cantidad de estudiantes como condición de la} gestión en el proceso de enseñanza-aprendizaje En el proceso de enseñanza-aprendizaje, la vinculación docente-estudiante es fundamental para promover aprendizajes significativos. Dicha relación puede hacerse más personal cuando existe una interacción entre docentes y grupos pequeños, tanto en el contexto aula como en la clínica.

En el contexto clínico, urge la necesidad de contar con mayor capacidad docente, para apoyar el proceso de aprendizaje de los estudiantes, en términos de seguridad, emocionalidad y resolución clínica técnica.

Esto es fundamental, ya que el docente debe resguardar todos los eventos que surgen en el contexto clínico, relacionados con el estudiante y con el equipo de salud, pues pueden ocurrir sucesos que deben ser regulados in situ.

\section{Formación docente en educación médica como eje principal en la calidad educativa}

Los docentes mencionan que tener formación en educación es esencial para comprender el proceso educativo. Si bien en algunos docentes 
existe resistencia a participar en programas de formación, quienes han buscado instancias para ello evalúan positivamente el tener conocimientos que los preparen para guiar el proceso de enseñanza-aprendizaje de sus estudiantes. Para ello, sería esencial que las instituciones cuenten con programas de formación docente inicial.

El comprender cómo planificar, desarrollar objetivos o resultados de aprendizaje, y diseñar actividades de enseñanza y evaluación, ayuda a los profesores a mejorar su docencia. Algunos académicos señalan que cuando iniciaron sus actividades docentes, utilizaban estrategias de modelos docentes que habían observado en su experiencia como estudiantes.

\section{Recursos económicos y espacio físico para ase- gurar calidad en educación}

Cuando las instituciones académicas inician un proceso de formación profesional en una disciplina del área de la salud, es fundamental contar con los recursos económicos necesarios para asegurar un proceso educativo de calidad, lo que se traduce en contar con espacios físicos necesarios para la carrera, materiales de enseñanza y una planta docente que pueda guiar el proceso educativo. Sin embargo, a veces existe poca sincronización entre espacios físicos, enseñanza, docente y objetivos educativos.

Al respecto, los docentes señalan como fundamental contar con espacios habilitados para realizar simulación clínica y laboratorios, como espacios de enseñanza para los estudiantes. Un docente manifiesta que, si bien existe apoyo importante por parte de la institución, éste no siempre se traduce en elementos operacionales que permitan a las carreras desarrollarse adecuadamente.

\section{Discusión}

Las carreras en Ciencias de la Salud se desarrollan en un contexto educativo complejo, en el cual se deben articular variados elementos para formar los profesionales que el país necesita ${ }^{1,9}$. Existen diversos estudios que abordan el fenómeno de la innovación educativa ${ }^{1,4,9,12-13,18-19,20-27}$ y los factores que interactúan en estos procesos de intervención. Sin embargo, pocos han concentrado sus esfuerzos en analizar aquellas condiciones que deben existir para impartir programas curriculares en carreras de la salud ${ }^{1,5}$. Así, este estudio permite dilucidar algunos aspectos claves a considerar en las instituciones de educación superior, lo cual es fundamental para reflexionar acerca de calidad en educación y cómo lograrla.

Considerando los resultados antes expuestos, se relevan ciertas condiciones mínimas para que los docentes puedan impartir una enseñanza de calidad en un contexto educativo en Ciencias de la Salud.

Las condiciones físicas del entorno educativo son esenciales para implementar nuevas metodologías de enseñanza, acorde a los modelos educativos adscritos por la institución. Es fundamental considerar si se promueven enfoques de enseñanza centrados en el profesor y en la transmisión de contenido $^{5,18}$. Si no existe un proceso reflexivo al respecto, habrá una distancia entre el discurso y la práctica institucional.

Paralelo a lo anterior, se ha consensuado la importancia de capacitar a los docentes universitarios en educación, estrategia clave para mejorar la efectividad docente, constituyendo un componente importante en la educación médica; pero sólo puede lograr cambios en el comportamiento del docente prestando atención a las concepciones de éste acerca de la enseñanza y aprendizaje $e^{8,9,19,20}$.

En este proceso formativo de competencias docentes, es fundamental considerar hasta qué nivel los docentes pueden ser autónomos en el proceso de enseñanza-aprendizaje. El docente puede tener mayor o menor autonomía sobre la cantidad de contenidos y la forma de enseñarlos, dependiendo de las políticas institucionales ${ }^{5,28,29}$ y esto se asocia también a los parámetros nacionales considerados por las agencias acreditadoras.

En este contexto, tampoco podemos dejar a un lado la cantidad de estudiantes atendidos por los docentes de carreras de la salud. Se reconoce que una de las prácticas pedagógicas más utilizada en las instituciones universitarias sigue siendo la clase magistral en cursos masivos ${ }^{21}$, cuya efectividad es dudosa pues con ella el alumno no es capaz de adquirir aprendizajes o conocimientos de manera autónoma ${ }^{22,25}$. Por esto, en Ciencias de la Salud se ha ido privilegiando el formato de clases en pequeños grupos ${ }^{26,27}$. Sin embargo, para ello es indispensable cautelar la proporción de cantidad de estudiantes por docente y la infraestructura necesaria, aspecto que generalmente escapa al control del docente siendo determinado a nivel 
institucional, sin considerar la realidad de las aulas, donde no existe relación con la cantidad de alumnos considerados en los procesos de selección. Así, tampoco se considera el efecto que dichos procesos de selección tienen en el actual sistema de regulación de campos clínicos, el cual regula la cohabitación con otras universidades en el mismo campo clínico y si el docente tiene contrato universitario o depende del Ministerio de Salud. Por consiguiente, si bien el rol del docente es un medio para el aprendizaje de los estudiantes $^{1,3}$ es fundamental que las instituciones brinden las condiciones necesarias para desempeñar una práctica educativa de calidad; de lo contrario, resulta ser anacrónico solicitar actualmente al docente cumplir con funciones en un contexto educativo que no está facilitando el proceso de enseñanza-aprendizaje.

Por lo anterior, para generar mejoras en el proceso de enseñanza-aprendizaje, resulta indispensable que las instituciones educativas consideren las condiciones necesarias para el contexto educativo de carreras de la salud y que ello no sea determinado a nivel central, contemplando únicamente aspectos económicos de sustentabilidad.

\section{Referencias}

1. Devlin M, Samarawickrema G. The criteria of effective teaching in a changing higher education context. Higher Education Research \& Development 2010; 29 (2): 11124.

2. Shim SH. A philosophical investigation of the role of teachers: A synthesis of Plato, Confucius, Buber, and Freire. Teaching and Teacher Education 2008; 24 (3): 515-35.

3. Alterio G, Ruiz C. Mediación metacognitiva, estrategias de enseñanza y procesos de pensamiento del docente de Medicina. Educ Med Super 2010; 24 (1): 25-32. Disponible en: http://scieloprueba.sld.cu [Consultado el 11 de agosto de 2017].

4. Gordon C, Debus R. Developing deep learning approaches and personal teaching efficacy within a preservice teacher education context. British Journal of Educational Psychology 2002; 72 (4): 483-511.

5. Badia A, Gómez F. Condiciones del contexto instruccional que afectan el diseño de la enseñanza del profesor universitario. Educación XX1 2014; 17 (2): 169-92. Disponible en: http://www.redalyc.org [Consultado el 11 de agosto de 2017].
6. Sanjuán Á, Martínez J. Nuevo enfoque en el proceso de enseñanza-aprendizaje para la interrelación de conocimientos y formación de clínica/comunitaria. Invest Educ Enferm 2008; 26 (2): 150-159. Disponible en: http://www.redalyc.org [Consultado el 11 de agosto de 2017].

7. Alzamora AM. El sentido de eficacia: un elemento de comprensión de la motivación docente. Revista de Educación 2001; 324: 297-316. Disponible en: http://www. mecd.gob.es/revista-de-educacion/ [Consultado el 11 de agosto de 2017].

8. Jacobs J, Muijtjens A, Van Luijk S, Van Der Vleuten C, Croiset G, Scheele F. Impact of institute and person variables on teachers' conceptions of learning and teaching. Medical Teacher 2015; 37 (8): 738-46.

9. Lindblom Ylänne S, Trigwell K, Nevgi A, Ashwin P. How approaches to teaching are affected by discipline and teaching context. Studies in Higher Education 2006; 31 (3): 285-98.

10. Bandura A. The assessment and predictive generality of self-percepts of efficacy. Journal of Behavior Therapy and Experimental Psychiatry 1982; 13 (2): 195-9.

11. Flexner A. The Flexner report on medical education in the United States and Canada. New York: Carnegie Foundation; 1910: 58.

12. Pernas M, Garí M, Arencibia L, Rivera N, Nogueira M. Consideraciones sobre las ciencias básicas biomédicas y el aprendizaje de la clínica en el perfeccionamiento curricular de la carrera de Medicina en Cuba. Educ Med Super 2012; 26 (2): 307-325. Disponible en: http://www. ems.sld.cu [Consultado el 11 de agosto de 2017].

13. Schauber S, Hecht M, Nouns Z, Dettmer S. On the role of biomedical knowledge in the acquisition of clinical knowledge. Medical Education 2013; 47 (12): 1223-35.

14. Schön D. El profesional reflexivo: Cómo piensan los profesionales cuando actúan. Barcelona, España: Editorial Paidós; 1998. 320 p.

15. Strauss A, Corbin J. Bases de la investigación cualitativa. Técnicas y procedimientos para desarrollar la teoría fundamentada. Medellín: Editorial Universidad de Antioquia; 2002.

16. Patton M. Qualitative evaluation methods. Beverly Hills, USA: Sage Publications; 1980. 381 p.

17. Vieytes R. Metodología de la investigación en organizaciones, mercado y sociedad. Buenos Aires, Argentina: Editorial de las Ciencias; 2004.745 p.

18. Jessop T, Gubby L, Smith A. Space frontiers for new pedagogies: a tale of constraints and possibilities. Studies in Higher Education 2012; 37 (2): 189-92.

19. Lyon Jr H, Holzer M, Reincke M, Brendel T, Ring J, 
Weindl A, et al. Improvements in teaching behavior at two German medical schools resulting from a modified Flanders interaction analysis feedback intervention process. Medical Teacher 2014; 36 (10): 903-11.

20. Steinert Y, Mann K, Centeno A, Dolmans D, Spencer J, Gelula $\mathrm{M}$, et al. A systematic review of faculty development initiatives designed to improve teaching effectiveness in medical education: BEME Guide $\mathrm{N}^{\circ} 8$. Medical Teacher 2006; 28 (6): 497-526.

21. Gehlen-Baum V, Weinberger A. Teaching, learning and media use in today's lectures. Computers in Human Behavior 2014; 37: 171-82.

22. Cantillon P. ABC of learning and teaching in medicine: teaching in large groups. BMJ 2003; 326: 437.

23. Cardozo S, Andino G, Brunnetti A, Espíndola E. Efectividad de los métodos activos como estrategia de enseñanza-aprendizaje en grupos grandes y heterogéneos. Educ Med Super 2008; 22 (1): 1-6. Disponible en: http://scieloprueba.sld.cu [Consultado el 11 de agosto de 2017].

24. Bowman N, Akcaoglu M. “I see smart people!”: Using Facebook to supplement cognitive and affective learning in the university mass lecture. The Internet and Higher Education 2014; 23: 1-8.

25. Isaza A. Clases magistrales versus actividades participativas en el pregrado de medicina. De la teoría a la evidencia. Rev Estud Soc 2005; 20: 83-91. Disponible en: http://www.scielo.org.co [Consultado el 11 de agosto de 2017].

26. Cendan J, Silver M, Ben-David K. Changing the Student Clerkship from Traditional Lectures to Small Group Case-Based Sessions Benefits the Student and The Faculty. Journal of Surgical Education 2011; 68 (2): 117-20.

27. Wolff M, Wagner MJ, Poznanski S, Schiller J, Santen S. Not Another Boring Lecture: Engaging Learners with Active Learning Techniques. The Journal of Emergency Medicine 2015; 48 (1): 85-93.

28. Prosser M, Trigwell K. Relations between perceptions of the teaching environment and approaches to teaching. British Journal of Educational Psychology 1997; 67 (1): 23-35.

29. Jones A. Generic attributes as exposed theory: The importance of context. Higher Education 2009; 58 (2): 175-91. 